\title{
Intense, Brilliant Micro $\gamma$-Beams in Nuclear Physics and Applications
}

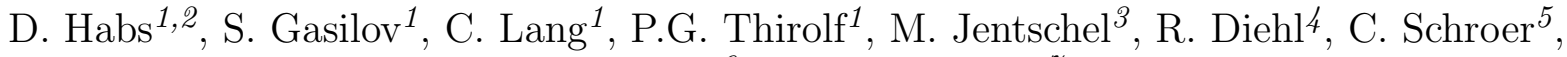 \\ C.P.J. Barty ${ }^{6}$ and N.V. Zamfir ${ }^{7}$ \\ ${ }^{1}$ Fakultät für Physik, Ludwig-Maximilians Universität München, D-85748 Garching, Germany \\ 2 Max-Planck-Institut für Quantenoptik, D-85748 Garching, Germany \\ 3 Institut Laue Langevin, 6 rue Jules Horowitz, F-38042 Grenoble Cedex 9, France \\ 4 Max-Planck-Institut für Extraterrestrische Physik, D-85748 Garching, Germany \\ ${ }^{5}$ Inst. for Structure Physics, TU Dresden, D-01062 Dresden, Germany \\ ${ }^{6}$ Lawrence Livermore National Laboratory, Livermore, CA 945500, USA \\ ${ }^{7}$ National Institute of Physics and Nuclear Engineering (IFIN-HH), Bucharest-Magurele \\ 077125, Romania
}

\begin{abstract}
The upcoming $\gamma$ facilities MEGa-Ray (Livermore) and ELI-NP (Bucharest) will have a $10^{5}$ times higher $\gamma$ flux $F_{0}=10^{13} / \mathrm{s}$ and a $\sim 30$ times smaller band width $\left(\Delta E_{\gamma} / E_{\gamma}=B W \approx 10^{-3}\right)$ than the presently best $\gamma$ beam facility. They will allow to extract a small $\gamma$ beam of about $30-100 \mu \mathrm{m}$ radius $1 \mathrm{~m}$ behind the $\gamma$ production point, containing the dominant $\gamma$ energy band width. One can collimate the $\gamma$ beam down to $\Theta_{B W}=\frac{\sqrt{B W}}{\gamma_{e}}$, where $\gamma_{e}=\frac{E_{e}}{m_{e} c^{2}}$ is a measure of the energy $E_{e}$ of the electron beam, from which the $\gamma$ beam is produced by Compton back-scattering. Due to the $\gamma$ energy - angle correlation, the angular collimation results at the same time in a reduction of the $\gamma$ beam band width without loss of "good" $\gamma$ quanta, however, the primary $\gamma$ flux $F_{0}$ is reduced to about $F_{\text {coll }} \approx F_{0} \cdot 1.5 \cdot \frac{\Delta E_{\gamma}}{E_{\gamma}}$. For $\gamma$ rays in the (0.1-100) MeV range, the negative real part $\delta$ of the index of refraction $n=1-\delta+i \beta$ from coherent Rayleigh scattering (virtual photo effect) dominates over the positive $\delta$ contributions from coherent virtual Compton scattering and coherent virtual pair creation scattering (Delbrück scattering). The very small absolute value $|\delta| \approx 10^{-6}-10^{-9}$ of the index of refraction of matter for hard X-rays and $\gamma$-rays and its negative sign - in contrast to usual optics - results in a very different $\gamma$-ray optics, e.g. focusing lenses become concave and we use stacks of $\mathrm{N}$ optimized lenses. It requires very small radii of curvature of the $\gamma$ lenses and thus very small $\gamma$ beam radii. This leads to a technical new solution, where the primary $\gamma$ beam is subdivided into $\mathrm{M} \gamma$ beamlets, which do not interfere with each other, but contribute with their independent intensities. We send the $\gamma$ beamlets into a two-dimensional array of closely packed cylindrical parabolic refractive lenses, where $N \approx 10^{3}$ lenses with very small radius of curvature are stacked behind each other, leading to contracted beam spots in one dimension. With a second $1 \mathrm{D}$ lens system turned by $90^{0}$, we can obtain small spots for each of the beamlets. While focusing the beamlets to a much smaller spot size, we can bend them effectively with micro wedges to e.g. parallel beamlets. We can monochromatize these $\gamma$ beamlets within the rocking curve of a common Laue crystal, using an additional angle selection by a collimator to reach a strongly reduced band width of $10^{-4}-10^{-6}$. We propose the use of a further lens/wedge arrays or Bragg reflection to superimpose the beamlets to a very small total $\gamma$ beam spot. Many experiments gain much from the high beam resolution and the smaller focal spot. This new $\gamma$ optics requires high resolution diagnostics, where we want to optimize the focusing, using very thin target wires of a specific nuclear resonance fluorescence (NRF) isotope to monitor the focusing for the resonance energy. With such beams we can explore new nuclear physics of higher excited states with larger level densities. New phenomena, like the transition from chaotic to regular nuclear motion, weakly-bound halo states or states decaying by tunneling can be studied. The higher level density also allows to probe parity violating nuclear forces more sensitively. This $\gamma$ optics improves many
\end{abstract}

Further author information: (Send correspondence to D. Habs)

D. Habs: E-mail: dietrich.habs@lmu.de, Telephone: 00498928914077

Harnessing Relativistic Plasma Waves as Novel Radiation Sources from Terahertz to X-Rays and Beyond II, edited by Dino A. Jaroszynski, Proc. of SPIE Vol. 8075, 807507 - @ 2011 SPIE · CCC code: 0277-786X/11/\$18 doi: $10.1117 / 12.891512$

Proc. of SPIE Vol. $8075807507-1$ 
applications, like a more brilliant positron source, a more brilliant neutron source, higher specific activity of medical radioisotopes or NRF micro-imaging.

Keywords: Gamma-ray sources, photonuclear reactions, gamma-ray lens

\section{INTRODUCTION}

We want to explore possibilites to combine future brilliant $\gamma$-beams produced by Compton backscattering from relativistic electrons with a new type of $\gamma$-ray optics. Since refractive solid X-ray lenses have been studied up to $200 \mathrm{keV}$ very successfully ${ }^{1}$ and proved to be very robust and reliable, we for the first time want to explore if the properties of these refractive lenses can be extended up to several MeV. The index of refraction $n$ can be decomposed into a real and an imaginary part $n=1-\delta+i \beta$. One expects, due to the Kramers-Kronig relation, ${ }^{2}$ that the additional absorptive processes of Compton scattering and pair creation also result in new contributions to the real part of the index of refraction. However, in the PhD thesis of J.S. Toll from $1952,{ }^{3}$ it is shown theoretically that up to $1000 \mathrm{MeV}$ the Rayleigh scattering - i.e. the virtual photoeffect contribution dominates the real part of the index of refraction:

$$
\delta=\frac{r_{e} \lambda_{\gamma}^{2}}{2 \pi} \rho_{m a t} \frac{Z}{A} \propto \frac{1}{E_{\gamma}^{2}}
$$

Here $r_{e}$ is the classical electron radius, $\lambda_{\gamma}=\frac{h c}{E_{\gamma}}$ is the $\gamma$ wavelength, $\rho_{\text {mat }}$ is the lens density in $\mathrm{g} / \mathrm{cm}^{3}$ and Z and $\mathrm{A}$ are the charge and mass number of the lens material, respectively. A is measured in grams. This real part $\delta$ becomes very small $\propto E_{\gamma}^{-2}$, and for a typical nuclear energy of $3 \mathrm{MeV}$ has a value of $\sim 10^{-9}$. This $\delta$ should be verified experimentally for such high $\gamma$ energies. On the other hand, this extension of refractive $\gamma$ lenses into the $\mathrm{MeV}$ range looks promising, once the new intense, brilliant $\gamma$ beams become available, going much beyond the hard X-ray beams $(\leq 200 \mathrm{keV})$ of synchrotron light sources.

In section 2 we describe present and future $\gamma$ beam facilities. In section 3 we discuss the new efficient $\gamma$ beam optics, which becomes possible for the very brilliant novel $\gamma$ beams using the concept of splitting up the $\gamma$ beam into many $\gamma$ beamlets. Converting the very small beam diameters into parallel beams allows for an effective use of a Laue crystal monochromator. ${ }^{4}$ Also the $\gamma$ beamlets can be used at distant target stations without loss of beam quality. In section 4 we discuss new possibilities with focused $\gamma$ beams in nuclear physics. In section 5 we show the much improved perspectives in different fields of application.

\section{2. $\gamma$ BEAMS}

Presently the most powerful $\gamma$-beam facility is the High-Intensity $\gamma$-ray Source (HI $\gamma \mathrm{S}$ ) at Duke University (USA). It was originally designed as a Free-Electron Laser (FEL). In a second step these FEL photons are backscattered from the relativistic electron bunches, resulting in $\gamma$ beams with (1-160) MeV of about $10^{8} \gamma / \mathrm{s}$ and a typical band width of $5 \% .{ }^{5}$ In Table 1 we compare the properties of $\mathrm{HI} \gamma \mathrm{S}$ with the MEGa-Ray facility, ${ }^{6}$ presently being constructed at the Lawrence Livermore National Laboratory (USA) for (0.5 -2.5) MeV $\gamma$-ray energies, and with the planned ELI-NP facility at Bucharest-Magurele, ${ }^{7}$ being an extended version of MEGa-Ray, reaching up to 19.6 MeV $\gamma$ energy. While MEGa-Ray should deliver $\gamma$ beams in 2013, the scheduled date for the ELI-NP $\gamma$ beam is 2015 .

The special setup of $\mathrm{HI} \gamma \mathrm{S}$ with the storage ring results in a rather large distance from the $\gamma$ production point to the target and correspondingly to a rather large $\gamma$-beam cross section radius at the target of about $17 \mathrm{~mm}$ for $5 \%$ band width. Comparing the properties of the $\gamma$-beams in Table 1, one finds a large "quantum" jump in the quality and the possibilities for the new Compton back-scattering $\gamma$ facilities MEGa-Ray and ELI-NP. The $\gamma$ energy of Compton-backscattering is given by:

$$
E_{\gamma}=\frac{4 \gamma_{e}^{2} \cdot E_{L}}{1+\left(\gamma_{e} \Theta\right)^{2}+\frac{4 \gamma_{e} E_{L}}{m_{e} c^{2}}}
$$


Table 1. Comparison of the $\gamma$ facilities HI $\gamma \mathrm{S}$ with MEGa-Ray and ELI-NP.

\begin{tabular}{|c|c|c|}
\hline property & $\overline{\mathrm{HI}} \gamma \mathrm{S}$ & $\begin{array}{c}\text { MEGa-Ray (M) } \\
\text { ELI-NP(E) }\end{array}$ \\
\hline flux $F_{0}$ & $10^{8} / \mathrm{s}$ & $10^{13} / \mathrm{s}$ \\
\hline$\gamma$ energy & $(1-160) \mathrm{MeV}$ & $(0.5-2.5) \mathrm{MeV}$ \\
\hline & & $(0.5-19.5) \mathrm{MeV}$ \\
\hline distance collision & $60 \mathrm{~m}$ & $\approx 1 \mathrm{~m}$ \\
\hline $\begin{array}{c}\text { point-target } \\
\Delta E_{\gamma} / E_{\gamma}\end{array}$ & $5 \cdot 10^{-2}$ & $10^{-3}$ \\
\hline
\end{tabular}

Here $E_{L}$ is the energy of the primary laser photons and we consider that the outcoming $\gamma$ photon has an angle $\Theta$ with respect to the electron beam direction. By using a collimator, which limits the angle $\Theta$ to an angle $\Theta_{B W}$ with

$$
\Theta_{B W}=\frac{\sqrt{B W}}{\gamma_{e}}
$$

we can limit the energy resolution $\Delta E_{\gamma} / E_{\gamma}$ to the band width BW without additional loss by narrowing down the opening angle. This is possible as the combination of the spreading due to the angular spread of the electron beam, as well as the energy spread of the electron beam and the laser beam are smaller than the shift of the $\gamma$ energy due to Eq. 2. The collimated intensity $F_{\text {coll }}$ is approximately given by: ${ }^{8}$

$$
F_{\text {coll }} \cong 1.5 F_{0} \frac{\Delta E_{\gamma}}{E_{\gamma}}
$$

where $F_{0}$ is the total $\gamma$ flux without collimation. By this collimation we gain in brilliance.

There is a second, more long-term development ongoing for even better $\gamma$ beams, using again laser Compton backscattering, but with an Energy Recovery Linac (ERL) for the electrons and a super-cavity for the laser, recycling the optical photons. At the KEK accelerator facility in Japan, an ERL project is presently pursued, aiming at a $\gamma$ beam with an intensity of $10^{13} s^{-1}$, but with a significantly better $\gamma$ beam energy resolution below $10^{-4} .9,10$ Here after a single interaction with the laser photons, the high energy electron bunches are re-injected into the superconducting electron linac with a phase, where they return most of their energy to the accelerator cavity and only very low-energy electron bunches are deposited in the beam dump. Thus always new electron bunches with very good energy spread und emittance are extracted from the photocathode, however, the energy and not the electrons themselves are recycled. One is aiming at an electron current of $100 \mathrm{~mA}$. In contrast to the concept of MEGa-Ray and ELI-NP, where one is using a normal-conducting electron linac with rather large electron bunch charges of $250 \mathrm{pC}$ at $120 \mathrm{kHz}$ in a burst mode, with a burst repetition rate of only $120 \mathrm{~Hz}$, for the ERL design on wants to use a CW operation with a much higher repetition rate of $1-10 \mathrm{GHz}$ and much smaller bunch charge of $\approx 10 \mathrm{pC}$. Due to the much smaller bunch charge, the energy and angle spread of the electron bunch is strongly reduced. Operating the accelerator cavities in a continuous mode without varying beam loading effects, the acceleration voltage can be stabilized much better. Thus a much smaller band width below $10^{-4}$ is expected, and by collimation to $\Theta_{B W}$, beams with much smaller opening angle and better energy resolution will be obtained. There are several other ERL projects under development. In Germany, a high-current and low-emittance demonstrator ERL facility (BERLinPro) is currently being developed at the Helmholtz-Zentrum Berlin. ${ }^{11}$ ERL technology has been pioneered at Cornell University together with the Thomas Jefferson National Laboratory. ${ }^{12-14}$

The improvement in the band width of the $\gamma$ beam to better than $10^{-4}$ would allow for a collimation to a much smaller angle $\Theta_{B W}$ without loss of "good" $\gamma$ quanta within the band width. We will discuss later that these micro $\gamma$-beams with much smaller beam diameter allow for a much more efficient application of $\gamma$ beam optical elements, because the corresponding division into even smaller $\gamma$ beamlets allows for much more efficient stronger focusing forces. It is essential that the primary flux $F_{0}$ is large enough, so that the collimated flux 


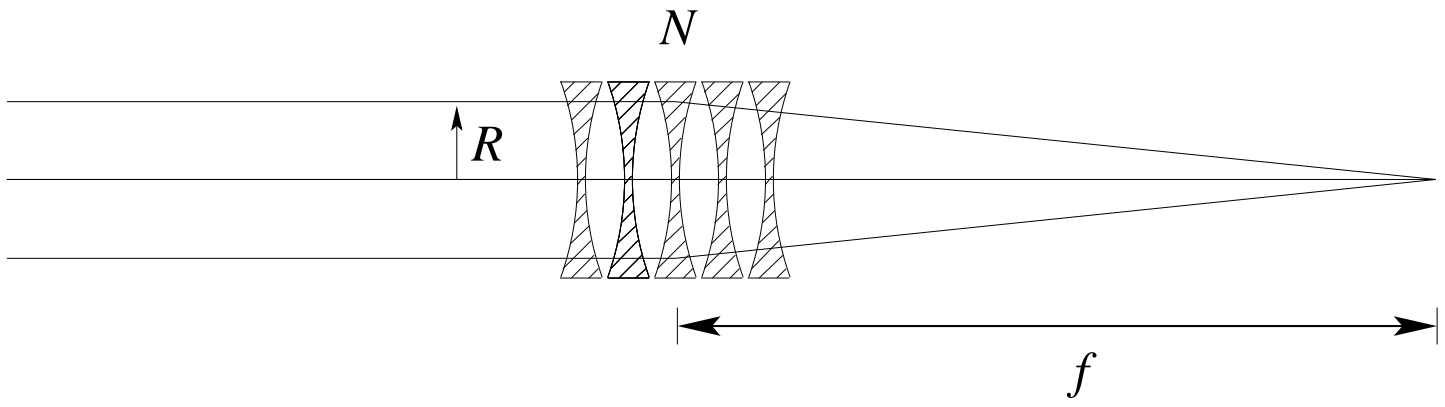

Figure 1. $\gamma$-lens consisting of $\mathrm{N}$ micro-lenses, irradiated with a $\gamma$-beam with radius $R$, comparable to the radius of the lens curvature $R_{L}$. The $\gamma$ lens has a focal length $f$.

$F_{\text {coll }}$ remains large enough to perform high-quality experiments. It seems possible to ultimately reach with ERL technology a primary flux larger than $10^{15} s^{-1}$ in an energy range of $(0.5-25) \mathrm{MeV}$.

Already now there exists a high quality $\gamma$ beam in the energy range of $(0.1-10) \mathrm{MeV}$ at the ILL reactor in Grenoble ${ }^{4}$ that can be employed to test the concepts of combining a $\gamma$ beam with new elements of $\gamma$ ray optics and learn about the feasibility of related experiments. Using the in-pile position at the high-flux reactor and samples with a large neutron capture cross section $(\geq 100 \mathrm{~b})$, an intense source of few times $10^{16} \gamma / \mathrm{s}$ is emitted isotropically. By placing a dedicated collimation system over $\sim 10 \mathrm{~m}$ from the target at the exits of a through-going beam tube, one obtains with $\sim 10^{7} \gamma / \mathrm{s}$ a spot diameter of $\sim 500 \mu \mathrm{m}$ and an opening angle of $\sim 50$ $\mu \mathrm{rad}$. The spectrum of the $\gamma$ rays is not tunable like in the Compton back-scattering production scheme. It is determined by the structure of the nucleus after neutron capture. There are typically many discrete transitions in the range of $0.1-100 \mathrm{MeV}$. The $\gamma$ lines usually have a thermal Doppler width of $\Delta E_{\gamma} / E_{\gamma} \sim 10^{-6}$. At the ILL it is possible to place crystal monochromators in the beam line to select individual capture transitions via Laue diffraction.

The $\gamma$ emission after neutron capture is not recoilless, like in Mössbauer spectroscopy. Thus a ground-state transition cannot be reabsorbed in Nuclear Resonance Fluorescense (NRF). However, this can be recovered in the case of light nuclei, for which strong $(n, \alpha+\gamma)$ reactions occur. Here a much larger Doppler broadening of the $\gamma$ rays due to the $\alpha$ recoil occurs, before stopping the recoil nucleus. We want to use the ${ }^{10} B(n, \alpha)$ reaction with a very large cross section of $3837 \mathrm{~b}$, leading to the $477.6 \mathrm{keV}$ E1 transition of ${ }^{7} \mathrm{Li}$ with a line width of $\sim 7.6$ $\mathrm{keV},{ }^{15}$ much larger than the Doppler shift due to the $477.6 \mathrm{keV}$ recoil. The $477.6 \mathrm{keV}$ level of ${ }^{7} \mathrm{Li}$ has a lifetime of 73 fs. Narrowing down the line width with crystal monochromators to $10^{-3}$ similar to MEGa-Ray, we expect a collimated intensity of $\sim 10^{6} \mathrm{\gamma} / \mathrm{s}$, which is $10^{4}$ times smaller than the $\Theta_{B W}$-limited MEGa-Ray beam intensity of $10^{10} \gamma / s$. Thus a $477.6 \mathrm{keV}{ }^{7} \mathrm{Li}$ experiment with NRF, as well as high-resolution focusing studies at higher $\gamma$ energies can be performed with an already existing $\gamma$ beam. Perhaps the envisioned $\gamma$ ray optics will allow to increase the $\gamma$ beam intensity at the ILL significantly. On the other hand, the $477.6 \mathrm{keV}$ transition of ${ }^{7} \mathrm{Li}$ has already been studied with NRF at T-REX, ${ }^{16}$ the precursor of the MEGaRray facility.

\section{3. $\gamma$ OPTICS}

We want to test the performance of the $\gamma$ optics with different components like refractive $\gamma$ lenses, monochromators and new diagnostics tools. Once the characteristics of the $\gamma$-ray optics have been established for the $477.6 \mathrm{keV}$ transition, we can proceed to higher $\gamma$-energy transitions, where the smaller real part of the index of refraction $\delta$ results in smaller focusing forces, requiring the use of more beamlets. Many high-accuracy techniques developped for $\gamma$ crystal spectrometers at the ILL may be taken over also for the large lens/wegde arrays of the new $\gamma$ ray optics.

\section{$3.1 \gamma$ lenses}

Let us start with the simplified picture of a $\gamma$ lens shown in Fig. 1. We have a $\gamma$ beamlet of radius $R$ and a stack of $\mathrm{N}$ thin lenses behind each other, which focus the parallel beam to a focus with focal length $f$. We use cylindrical 


\section{photons in silicon}
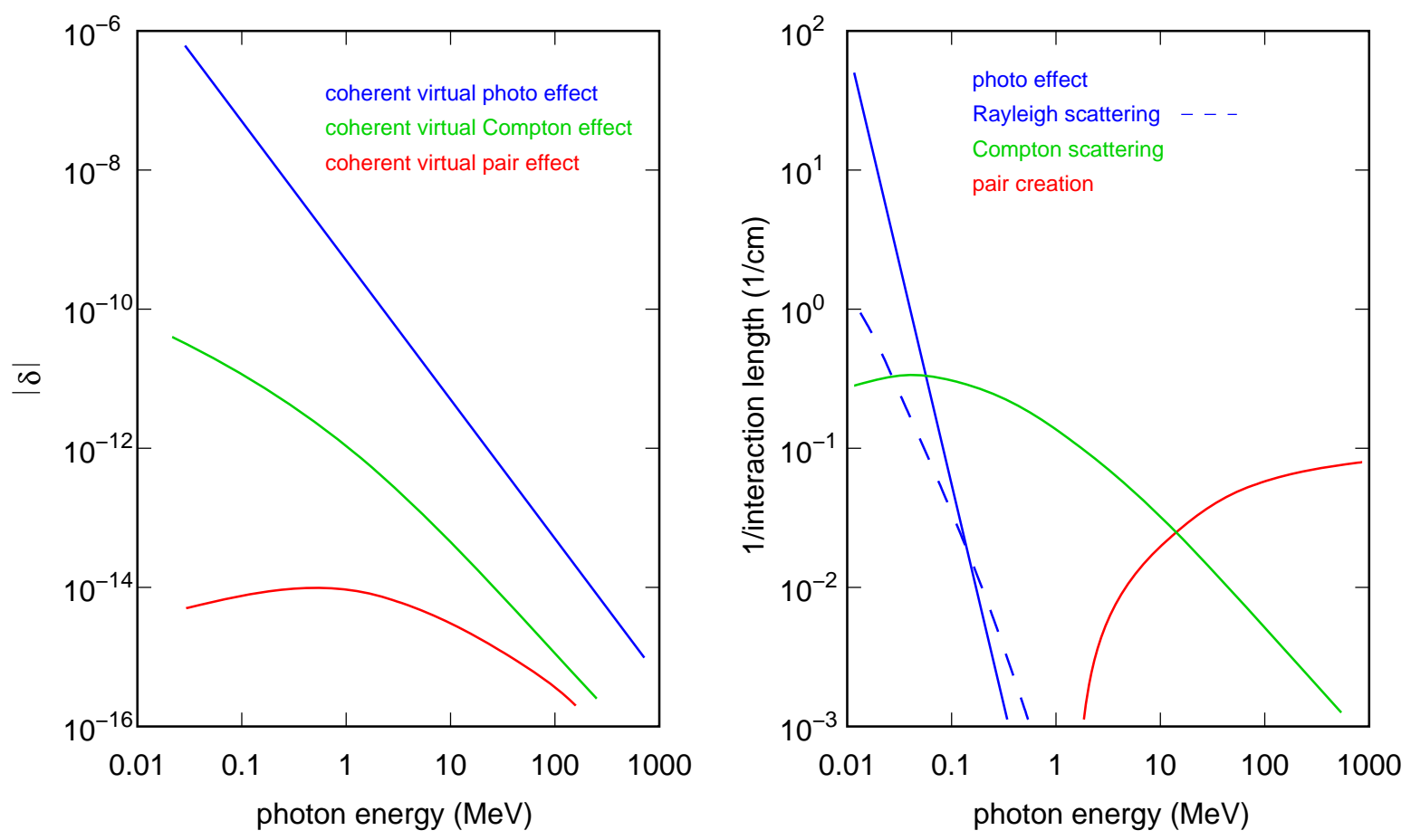

Figure 2. Real part $\delta$ of the index of refraction and absorption length $d_{1 / 2}$ for silicon and photons between $10 \mathrm{keV}$ and $1000 \mathrm{MeV}$. With the known real and imaginary part of the index of refraction we can optimize Si array optics for high transmittivity and high resolution.

parabolic lenses in the $1 \mathrm{D}$ case or spherical parabolic lenses in the $2 \mathrm{D}$ case, ${ }^{18,19}$ which are characterized by a central radius of curvature $R_{L}$ with

$$
|z|=\frac{y^{2}}{2 R_{L}} ;|z|=\frac{\rho^{2}}{2 R_{L}}
$$

The focal length $f$ of the lens is given by

$$
f=\frac{R_{L}}{2 \delta N}
$$

where $\delta$ is the small real part of the index of refraction and $N$ is the number of micro lenses. Since $\delta$ decreases with $E_{\gamma}^{-2}$, the realization of sufficent focusing strength becomes more difficult for higher $\gamma$ energies. A second important design consideration is the absorption within the lens, where $d_{1 / 2}$ is the half-thickness of the absorbing lens material being proportional to $1 / \rho_{\text {mat }}$. Since $\delta$ is proportional to $\rho_{\text {mat }}$, we can keep the focal length $f$ constant by compensating a larger $\rho_{\text {mat }}$ by a proportionally smaller number $N$ of micro lenses. Only with the new highly-brilliant $\gamma$ beams, the smaller beam radius $R$ allows to use a smaller lens curvature radius $R_{L}$, partially compensating the smaller $\delta$ for higher $\gamma$ energies to obtain reasonable focal lengths $f$. A further step to reduce the focal length is to stack a larger number $N$ of micro-lenses behind each other. It is important that the central regions of the micro lenses absorb very little of the $\gamma$ beam.

Since these micro lenses can be manufactured best from silicon, we show in Fig. 2 the deviation $\delta$ from the index of refraction and the absorption length $d_{1 / 2}$ for silicon as a function of $\gamma$ energy. In Ref. ${ }^{3}$ the coherent scattering amplitude for forward scattering has been calculated for all basic processes: Rayleigh scattering via the virtual photo effect, Delbrück scattering via virtual pair creation, Compton forward-scattering amplitude via the virtual Compton effect and nuclear Thompson scattering. Since the real part of the index of refraction so far has not been experimentally studied above $200 \mathrm{keV},{ }^{20,21}$ we will investigate it up to about $8 \mathrm{MeV}$ for the first time. 
X-ray solid refractive lenses have been used very successfully since $1996 .{ }^{22}$ Many new techniques have been developped. Using silicon, with electron-beam lithography and deep reactive ion etching, large arrays of nanofocusing lenses can be produced. ${ }^{23}$ Adiabatic focusing lenses allow to obtain much smaller $\gamma$ beam spots. ${ }^{24}$ At the ESRF, a tunable X-ray focusing lens system - called transfocator - allows to keep a fixed focal length for different $\mathrm{X}$-ray energies by remotely varying the number $N$ of lenses. The transfocator also acts as a monochromator and significantly enlarges the focused intensity. ${ }^{1}$

\subsection{1 $\gamma$-ray crystal reflection and beam monochromatization}

More than 20 years experience exist at the research reactor of the Institut Laue-Langevin in Grenoble with its $\gamma$ beam in measuring nuclear transition energies with the world's highest resolution of $10^{-6}$ and an accuracy of $10^{-8}$ using double crystal spectrometers called GAMS. ${ }^{4,17,28,29}$ Presently the most recent version GAMS6 is taken into operation, where many arts of metrology are combined to reach this extremely high stability and reproducibility. All these techniques become also relevant when applying them to brilliant $\gamma$ beams.

The basic relation for Laue-crystral spectroscopy is rather simple:

$$
2 d \sin \theta_{n}=n \lambda_{\gamma}=n \frac{h c}{E_{\gamma}}
$$

Here $n$ is the diffraction order, $\theta_{n}$ the according diffraction angle, $d$ the lattice spacing of the diffracting crystals and $\lambda_{\gamma}=h c / E_{\gamma}$ the wavelength of the gamma rays. The measurement is based on the knowledge of the lattice spacing $d$, which is assured by combined optical and X-ray interferometry to $\Delta d / d \simeq 10^{-9} \cdot{ }^{25}$ The measurement of the diffraction angle itself requires (1) the physical definition of the angle and (2) the capability to measure it. The definition of the angle (orientation of two axes with respect to each other) cannot be realized by pure collimation. In the case of the GAMS spectrometers this is done by the first crystal, which due to its very narrow acceptance angle diffracts photons only within a very narrow range of a few nrad towards the second crystal. The second crystal diffracts from this well-defined beam and the resulting angle is measured. For the measurement itself optical angle interferometers are used. These devices transfer excellent performances of heterodyne displacement interferometry (typically $10 \mathrm{pm}$ resolution over a meter of range) to a few picoradian resolution.

For monochromatization via crystal diffraction we can express the approximate energy dependence of the angle:

$$
\theta_{n}=n \frac{h c}{2 d E_{\gamma}}
$$

It is worth noting that the diffraction angle scales with $\sim 1 / E_{\gamma}$. The single crystal rocking curve of a perfect crystal can be calculated via dynamical diffraction theory ${ }^{26}$ and it can be shown that the full width at half maximum (FWHM) also scales with $\sim 1 / E_{\gamma}$. For a typical crystal lattice spacing $d_{220}=192015.6 \mathrm{fm}$ for Si220 and a wavelength of $1200 \mathrm{fm}$ for a $1.0 \mathrm{MeV} \gamma$ ray, we obtain a Bragg angle of about 6 mrad. The FWHM of the single crystal rocking curve has a value of about $10 \mathrm{nrad}$, decreasing with order $\mathrm{n}$, independent of the $\gamma$ energy. From this it can be concluded that a resolution of $\Delta E_{\gamma} / E_{\gamma} \sim 10^{-6}$ can be obtained.

\subsubsection{A possible scheme of $\gamma$ ray optics in combination with brilliant $\gamma$ beams using $\gamma$ beamlets}

Fig. 3 illustrates the main elements of the new $\gamma$-ray optics, the central issue of this contribution. For the given opening angle of the $\gamma$ beam and the closest usable distance $f$ from the production point, one obtains a radius $R$ of the $\gamma$ beam. We first could think of Fresnel lenses with a kinoform profile ${ }^{24,27}$ with a step function in the profile becoming very closely spaced and very difficult to produce in the outer regions of the lens. Furthermore, the length $\lambda_{\gamma} / \delta$ for a phase shift of $2 \pi$ becomes for $\gamma$ rays rather large and the parabolic approximation for the lens is fully sufficient. Thus we prefer to subdivide the $\gamma$ beam into a number of $\gamma$ beamlets, which no longer interfere with each other, but the local Fresnel lens, a parabolic lens, for each beamlet is very easy to produce. We later can add the intensities of the $\gamma$ beamlets into a common focus. Since the $\gamma$ rays are produced incoherently and since the direction of the emitting recoiling particle can be measured, in principle the direction of the $\gamma$ 

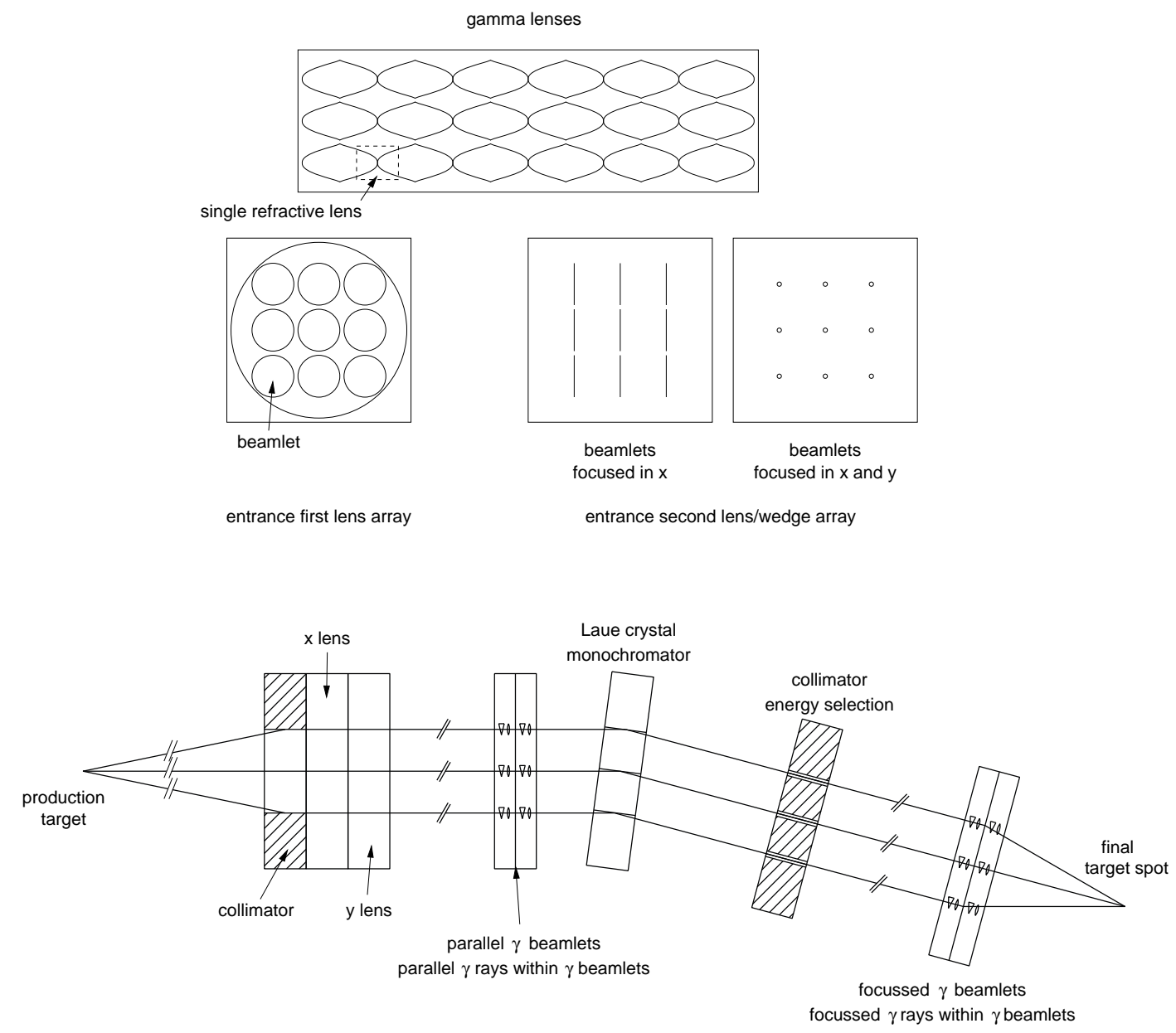

Figure 3. Example of a $\gamma$ beam combined with $\gamma$ optics. The first lens array results in a contraction of the $\gamma$ beamlets. Then a second lens/wedge array tilts the $\gamma$ beamlets to make then all parallel and at the same time the lenses produce parallel $\gamma$ rays within each beamlet. After the energy selection with the Laue crystal and the collimator, a further lens/wedge array combines all $\gamma$ beamlets into a final target spot. 
ray will be limited to its beamlet with respect to quantum mechanical interference. Thus the use of beamlets is very natural. After contracting each beamlet to a much smaller size with focusing lenses, the following optical manipulations can be performed with much smaller elements with larger focusing forces. Then a really flexible treatment of the many beamlets allows for a much improved $\gamma$ optics. Thus for the $477.6 \mathrm{keV}{ }^{7} \mathrm{Li}$ transition we plan for 9 beamlets, closely packed with each beamlet having a typical radius or $30 \mu \mathrm{m}$. Each beamlet is focused by a stack of $N \approx 2000$ thin parabolic lenses. The estimated length of such a lens array is $5 \mathrm{~cm}$. In Fig. 3 we show in the upper part these lenses, which can be produced from silicon using existing industrial developments. After transfering the mask by electron-beam lithography to the $\mathrm{Si}$ wafer, the deep lens structures are obtained by reactive ion etching. ${ }^{23}$ Here we use one central layer of silicon with 3 beamlets and an upper and lower layer produced with a separate mask. The aim is to contract the beamlets adiabatically ${ }^{24}$ to some smaller diameter, e.g. being reduced 10-fold and thus gaining sufficient deflecting power with some added micro-wedges to deflect the outer beamlets to become parallel with respect to the central beamlet. Thus the originally diverging beamlets can be bent into parallel beamlets, or one could even go further and change them into converging beamlets.

At first sight it seems to contradict the conservation of the $\gamma$ beam emittance that the large diameter beamlets with diverging rays can be transformed into much smaller diameter beamlets with parallel rays, however, the initial emittance of the larger diameter beamlet is that small, that the resulting smaller diameter beamlet indeed exhibits a reduced, but still sufficient parallelity. The beamlets originate as very small cones from the very small diameter production point and one coulds imagine a very small lens which transforms it into a vey small diameter beamlet, thus demonstrating the excellent emittance.

We will use 1D cylindical lenses that can easily be manufactured. Thus the lens will focus in $\mathrm{x}$-direction, producing line foci of the beamlets. A second lens system turned by $90^{0}$ would focus in y direction, resulting in a beam diameter contracted by a factor of $\approx 10$ for each beamlet after some length 1 , as shown in Fig. 3. These smaller diameter beamlets can now be transformed into parallel beamlets by small wedges and by small defocusing lenses with much smaller focal lengths and larger radii of curvature, thus being transformed into beamlets with parallel $\gamma$ rays within each beamlet. For both primary focusing lenses together, we expect for each beamlet a transmission of more than 50\%. We want to send these contracted parallel beamlets to a common $\mathrm{Si}[220]$ crystal for monochromatization. This is similar to the use in the double crystal spectrometer GAMS, where the first crystal defines a beam direction and the second crystal leads to the energy dispersion. Here we replace the first crystal by the lens/wedge array. With the same techniques developped for the double crystal spectrometer, we now have to measure very accurately the relative angle between the lens/wedge array and the Laue crystal. After some distance from the Laue crystal, we select with a collimator the opening angle of the beamlets to obtain the proper energy spread. This possibility to monochromatize a $\gamma$ beam independent from its production mechanism ranging from a primary bandwidth of $10^{-3}$ to a much smaller value down to $10^{-6}$ is very essential for high resolution nuclear spectroscopy. Here we use the achromatic properties of both the refractive lens array and the Laue crystal. With the $477.6 \mathrm{keV}{ }^{7} \mathrm{Li}$ transition we want to gain experience in the alignment and tuning properties of this system. We will develop targets with small LiF structures to map out the focal properties for the resonance energy by NRF.

In a final step the beamlets can be superimposed by a further lens/wedge array to a common small focus. Here also a set of Bragg crystals could be used, but the lense/wedge array, using solid $\gamma$ optics seems more easy to be build. Such a focus will have a much larger $\gamma$ beam intensity. Once this more efficient manipulation of contracted beamlets has been proven, it will become a very useful principle even for a much larger number of beamlets to build lenses for even higher $\gamma$ energies with larger numerical apertures.

We have to consider the special case that we will always use $\gamma$-beams produced by Compton back-scattering from relativistic electrons in combination with the $\gamma$-lens and make use of the very special $\gamma$ energy-angle correlation of Compton backscattered $\gamma$ rays to work with a very small radius $R$ resulting in a natural monochromatization. The outer angles $\Theta$ are no longer focused by the lens. Thus the first collimator has not to be a very tight one, because also the lens array acts as collimator. We can experimentally monitor the energy range focused by the lens by using a special nuclear resonance $\gamma$ energy and measure the nuclear resonance fluorescence (NRF) for a thin wire target, probing the $\gamma$ beam focus behind the $\gamma$ lens. The better the bandwidth of the central $\gamma$ beam, the smaller the radius $R$ and the focal length $f$ will be. For higher $\gamma$ beam energies and correspondingly larger $\gamma_{e}$, the radius $R$ will be smaller, partially compensating the strong $\gamma$ energy dependence of the focal length $f$ 
with $\propto \frac{1}{E_{\gamma}^{2}} \propto \frac{1}{\gamma_{e}^{4}}$. By increasing the number $N$ of micro-lenses, we can keep the focal length sufficiently small for $\gamma$ beams with sufficiently good primary bandwidth BW. In the very central region, the very thin micro-lenses will lead to a small absorption of the $\gamma$ beam. We have to align the $\gamma$ lens very accurately with respect to the electron beam direction, but again this can be monitored by NRF scattering measurements.

The strong decrease of the real part of the refractive index $\delta$ with $\gamma$ energy is partially compensated by the improved opening angle of the $\gamma$ for higher $\gamma$ energies, resulting in an only linear increase of the focal length with $\gamma$ energy. Still, we will need for each $\gamma$ energy a dedicated lens array.

\section{NEW NUCLEAR PHYSICS}

Here we list many examples where basic nuclear physics will profit from the much improved properties of $\gamma$ beams after merging them with $\gamma$ beam optics.

\subsection{High resolution spectroscopy}

One interesting aspect of high resolution nuclear spectroscopy is the measurement of weak parity-violating nuclear forces. In perturbation theory, the mixing of parity doublets is strongly enhanced for closely-spaced parity doublets, because in calculating the mixing amplitude, the mixing matrix element is divided by the small energy difference between the levels of opposite parity. ${ }^{30}$ With increasing excitation energy, we can move from level spacings of $\mathrm{keV}$ as considered in Ref. ${ }^{30}$ to $\mathrm{eV}$ spacings, allowing for a much better determination of the theoretically predicted mixing matrix elements. ${ }^{31}$ Also for resonances just above the neutron binding energy, strong parity nonconservation effects have been observed. ${ }^{32}$

With the high resolution we can also measure the widths of nuclear levels after $\gamma$ excitation and determine the lifetime of levels in the fs-range by measuring if the decay occurred in flight or after stopping in the target by measuring the Doppler broadening of the $\gamma$ transitions, induced by the exciting $\gamma$ recoil. ${ }^{33,34}$ In contrast to former measurements, where levels were populated in $(n, \gamma)$ cascades, here we can study different nuclei in $\left(\gamma, \gamma^{\prime}\right)$ cascades.

\subsection{Spectroscopy of highly excited states}

Though recently rather reliable equations and models like the back-shifted Fermi gas model or the constanttemperature model have been developped, ${ }^{36,37}$ here we are more interested in the fluctuating properties of highly excited levels. In this context Wigner and Dyson ${ }^{38}$ started to develop the random-matrix theory. This development culminated in recent reviews by H.A. Weidenmüller et al., ${ }^{39,40}$ which allows to study quantum chaos in nuclei. With the new possibilities of very high resolution photoexcitation studies, we can study fluctuating quantities of highly excited levels, like nearest-neighbour level energy spacing. We will study this as a function of excitation energy to observe the transition from chaotic to regular nuclear motion.

\subsection{Spectroscopy of weakly bound states}

While weakly bound halo nuclear states have been studied for very neutron-rich nuclei, ${ }^{41,42}$ in our case we are interested to study similar states for nuclei with a stable ground state, where we excite halo s-wave neutron states by photoexcitation just below the neutron binding energy. Here again the much better energy resolution of $\gamma$ beams achievable with the described new $\gamma$ optics would make such measurements possible. This identification and measurements of lifetimes of such neutron halo states would have significant relevance for the new neutron sources discussed below.

\subsection{Spectroscopy of tunneling states}

Actinide nuclei show a very complex nuclear potential landscape with strongly deformed second and third minima, being caused by shell effects for rather regular nuclear motion, when the nuclear axis ratio is close to 2:1 or 3:1. We could probe these configurations by populating resonances in these minima with $\gamma$ beams of very narrow band width. We would study the following fission decay of these resonances. As a starting case ${ }^{238} \mathrm{U}$ appears very suitable, because we know the properties of neighbouring nuclei quite well and have good predictions for these potential minima. ${ }^{43}$ 


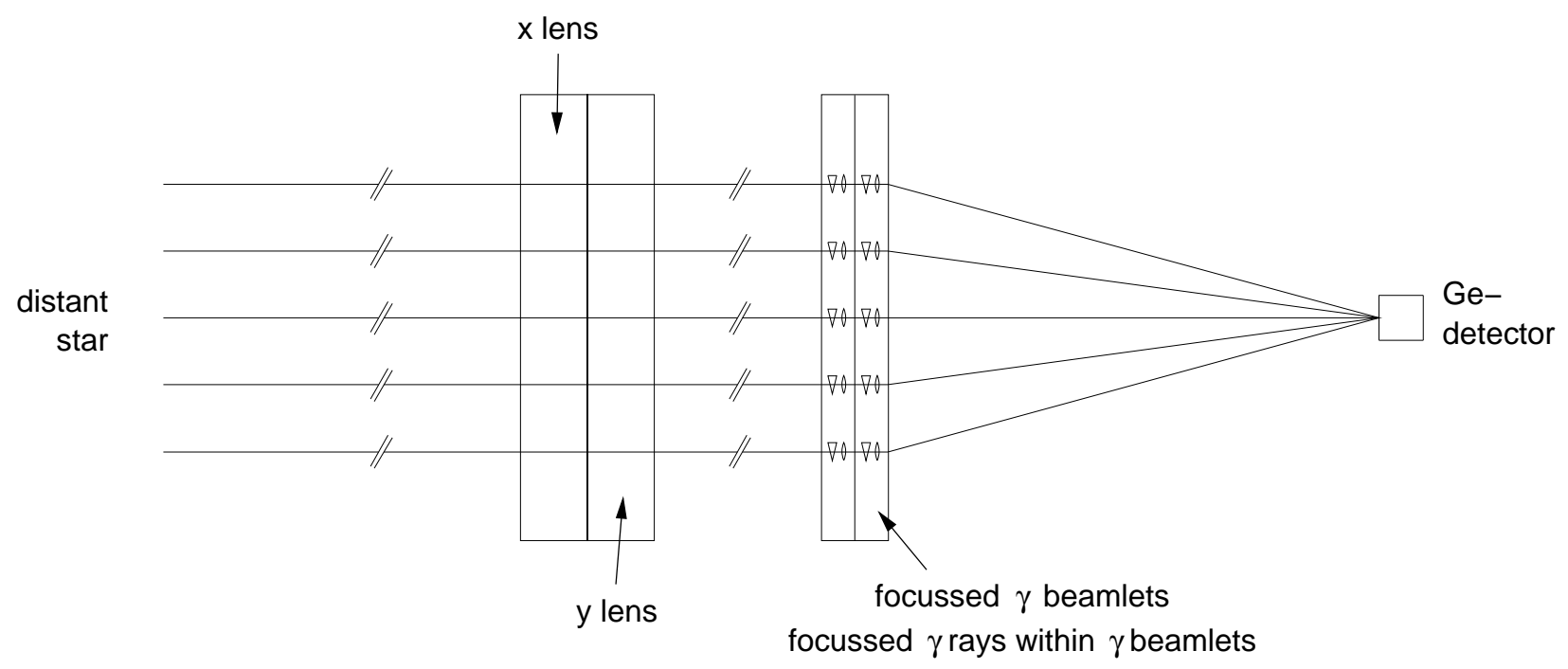

Figure 4. Focusing a much higher flux of a $\gamma$ line from a distant star with the new $\gamma$ optics into a Ge detector.

\section{APPLICATIONS}

In a similar way we list several applications, where the combination of brilliant, intense $\gamma$ beams with $\gamma$ optics results in important improvements.

\subsection{Flux concentrators for astrophysics or an ILL $\gamma$ beam}

Such $\gamma$ micro beamlets can also be used to optimize astrophysics experiments: We have some extremely distant $\gamma$ ray source at typically $5 \cdot 10^{21} \mathrm{~cm}$ distance with some typical intensity of $10^{-5} / \mathrm{cm}^{2} \mathrm{~s}$ ). We want to enlarge the area of detection by concentrating the many beamlets $\mathrm{M}$ with reduced beamlet diameters after focusing them with stacks of many lenses $\mathrm{N}$ and by guiding them with strong focusing forces and deflecing wedges into a much smaller area. Thus we can improve the counting rate significantly and at the same time use the monochromatization of the $\gamma$ ray optics. Interesting transitions would be the $511 \mathrm{keV}$ annihilation radiation, the $1157 \mathrm{keV}$ transition of ${ }^{44} \mathrm{Ti}$ or the $1809 \mathrm{keV}$ transition of ${ }^{26} \mathrm{Al}$ from supernova remnants and the $847 \mathrm{keV}$ transition from ${ }^{56} \mathrm{Ni}$. Such measurements could even be extended to measure the Doppler broadening and temperatures in astrophysical processes, if we could reach sufficiently good efficiencies to contract and monochromatize very many parallel beamlets originating from astrophysical objects (like the $1157 \mathrm{keV}$ transition of ${ }^{44} \mathrm{Ca}$ following the 60 year decay of $\left.{ }^{44} \mathrm{Ti}^{35}\right)$. Here the task of MEGa-Ray would be to optimize much larger $\gamma$ lens arrays, which later could be used on satellite missions in $\gamma$-ray astronomy.

In a similar way we could use a much larger solid angle from the isotropic emitting $\gamma$ source of the ILL reactor by transforming a rather monochromatic $\gamma$ transition with a typical thermal line width of $10^{-6}$ with a large array of $\gamma$ beamlets into a small diameter $\gamma$ beam with several orders of magnitude higher intensity than the present $\gamma$ beam.

\subsection{Imaging very localized $\gamma$ sources in nuclear medicine}

We want to test the $\gamma$ ray optics to image $\gamma$ ray sources with much better resolution using this phase-sensitive wave optics. Preferentially we should select nuclear transitions with energies around $100 \mathrm{keV}$ (similar to SPECT transitions) to increase $\delta$ and the focusing power. With our optics with small-diameter beamlets, we could even produce enlarged images. The independent pictures of the beamlets, similar to an insect facet eye, could be used to increase the collection efficency. Thus we could achieve images of small tumors with $\mu \mathrm{m}$ resolution, while present SPECT cameras have only $\mathrm{mm}$ resolution. One could also study the images of lower energy transitions $\left(495 \mathrm{keV},{ }^{17} \mathrm{~F}\right)$ produced in ion irradiation of cancer therapy to monitor the range and track of ion radiation. Again the use of many beamlets can increase the detection efficiency. 


\subsection{Medical Radioisotopes}

We have described in detail that many new medical radioisotopes can be produced by photoexcitation and photonuclear reactions. ${ }^{45,46}$ Again the much smaller $\gamma$ beam diameter allows to reach higher specific activities and to use frequently very small enriched targets. A high specific activity is very essential for medical radioisotopes, because the transporting bioconjugates, which reach the essential docking molecules, do not carry nonradioactive or homolog chemical elements.

\subsection{A brilliant positron source}

We recently showed that a narrow $\gamma$ beam of (2.0-2.5) $\mathrm{MeV}$ allows for a much higher conversion efficiency to obtain moderated positrons from high $\mathrm{Z}$ materials than a source of isotropically emitted $\gamma$ quanta. ${ }^{44}$ Thus the much more contracted $\gamma$ beams, which become possible through the $\gamma$ lenses, will result in even better conversion efficiencies. Thus we expect that with the MEGa-Ray or ELI-NP facilities the worldwide most intense and most brilliant positron sources can be produced.

\subsection{Nuclear resonance fluourescence with micro $\gamma$ beams}

Nuclear resonance fluorescence (NRF) allows to detect specific isotopes. Here one main aim is to detect actinides for radioactive waste management ${ }^{47}$ or to detect hidden isotopes in the context of safety assays. The big advantage of $\gamma$ beams is that they exhibit a very high penetrability. With the ability to produce beams with much smaller diameter, new micro-beam applications become possible, similar to X-ray beams, ${ }^{48}$ however having an isotope sensitivity and not a chemical element sensitivity and much deeper penetration.

\subsection{Brilliant micro neutron source}

We have proposed a new possibility to produce a brilliant, intense micro neutron beam by first transfering the neutrons into weakly bound halo states and then, after the isomers have stopped in the target and lost their recoil energy, the neutrons are released by a second photon beam. ${ }^{49}$ Again the possibility to produce much smaller neutron sources with much less background using the $\gamma$-ray optics results in more brilliant micro-neutron beams.

\section{CONCLUSION}

The combination of intense, brilliant $\gamma$ beams with $\gamma$ ray optics, using well established techniques from hard Xrays or crystal spectrometers looks promising, but has to be proven by experiment. It will require high-precision alignment and much improved high-resolution diagnostics using NRF. We can optimize $\gamma$ ray optics for detector systems with much improved energy resolution, spatial resolution and efficiency.

\section{REFERENCES}

[1] I. Snigireva et al., AIP Conf. Proc. 705, 708 (2004).

[2] J.D. Jackson, Classical Electrodynamics, sect 7.10, John Wiley, New York, (1975).

[3] J.S. Toll, PhD thesis, The dispersion relation of light and its application to problems involving electron pairs, Princeton, 1952.

[4] Deway et al., Phys. Rev. C 73, 044303 (2006).

[5] H.R. Weller, M.W. Ahmed, H. Gao, W. Torrow, U.K. Wu, M. Gai, R. Miskimen, Research opportunities at the upgraded HI $\mathrm{S}$ facility, Prog. Part. Nucl. Phys. 62, 257 (2009).

[6] C.P.J. Barty, Development of Mega-Ray technology at LLNL, http://www.eli-np.ro/documents/eli-excommeeting/ Barty-100412-ELI-NP-LLNL-MEGa-ray-Intro.pdf (2010).

[7] ELI-NP http://www.eli-np.ro/

[8] V.N. Litvinenko, Nucl. Instr. Meth. A 507, 527 (2003).

[9] R. Hajima, N. Nakamura, S. Sakanage, Y. Kobayashi, Design study of the compact ERL, KEK-Report 2007-7, JAEA-Research 2008-032 (2008). 
[10] T. Hayakawa, N. Kikuzawa, R. Hajima, T. Shizuma, N. Nishimori, M. Fujiwara M. Seya, Nondestructive assay of plutonium and minor actinides in spent fuel using nuclear resonance fluorescence with laser Compton scattering [gamma]-rays, Nucl. Instr. Meth. A 621, 695 (2010).

[11] A. Jankowiak, M. Abo-Bakr, W. Anders, T. Kamps, J. Knobloch, B. Kuske, O. Kugeler, A. Matveenko, A. Meseck, A. Neumann, T. Quast, J. Rudolph, BERLinPro - a compact demonstrator ERL for high current and low emittance beams, Proceedings of LINAC 10, Tsukuba, Japan, Sept. 12-17, 2010, to appear. on http://www.JACoW.org/.

[12] D.H. Bilderback, J.D. Brock, D.S. Dale, K.D. Finkelstein, M.A. Pfeifer, and S.M. Gruner, Energy recovery linac (erl) coherent hard $x$-ray sources, New Journal of Physics 12, 035011 (2010), http://stacks.iop.org/1367$2630 / 12 / \mathrm{i}=3 / \mathrm{a}=035011$.

[13] D.H. Bilderback, G. Hoffstaetter, S.M. Gruner, R\&D towards an energy recovery linac at synchrotron light sources, Synchrotron Radiation News 6, 32 (2010).

[14] M. Liepe, S. Belomestnykh, E. Chojnacki, Z. Conway, G. Hoffstaetter, R. Kaplan, S. Posen, P. Quigley, J. Sears, V. Shemelin, V. Veshcherevich, Latest results and test plans from the $100 \mathrm{~mA}$ Cornell ERL injector SCRF cryomodule, Proceedings of IPAC 10, Kyoto, Japan, May 23-28, 2010, http://accelconf.web.cern.ch/AccelConf/IPAC10/papers/wepec066.pdf

[15] C. Hofmeyr, Nucl. Instr. Meth. B 35, 435 (1998).

[16] T. Albert et al., Opt. Lett. 35,354 (2010).

[17] J. Krempel, A new spectrometer to measure the molar Planck constant, Thesis, LMU Munich, (2010).

[18] A. Snigirev et al, Appl. Opt. 47, 653 (1998).

[19] B.X. Yang, Nucl. Instr. Meth. A 328578 (1993).

[20] C.T. Chantler, J. Phy. Chem. Ref. Data Vol. 29, No. 4, 2000.

[21] M. Deutsch, Phys. Rev. B 30, 640 (1984).

[22] A. Snigirev et al., Nature 384, 49 (1996).

[23] C.G. Schroer et al., Appl. Phys. Lett. 82, 1485 (2003).

[24] C.G. Schroer and B. Lengeler, Phys. Rev. Lett. 94, 054802 (2005).

[25] E. Massa et al., J. Appl. Cryst. 43, 293-296 (2010).

[26] W.H. Zachariasen, Theory of X-ray diffraction in crystals, J. Wiley and Sons (1945).

[27] A.F. Isakovic et al., J. Synch. Rad. 19, 8 (2009).

[28] S. Rainville et al., Nature 438, 1096 (2005).

[29] G. Basile et al., Phys. Rev. Lett. 72, 3133 (1994).

[30] A.I. Titov et al., J. Phys. G: Nucl. Part. Phys. 32, 1097 (2006).

[31] E. Adelberger et al., Ann. Rev. Nucl. Part. A 316, 501 (1985).

[32] V.P. Alfimenko et al., Nucl. Phys. A 398, 93 (1983).

[33] T. Kahn et al., Nucl. Instr. Meth. A 385, 100 (1997).

[34] M. Jentschel et al., Nucl. Instr. Meth. B 115, 446 (1996).

[35] V. Schönfelder et al., AIP Conf. Proc. CP510, 54 (2000).

[36] T.v. Egidy and D. Bucurescu, Phys. Rev. C 80, 093310 (2010).

[37] T.v. Egidy and D. Bucurescu, Phys. Rev. C72, 044311 (2005).

[38] C.F. Porter; Statistical Theories of Spectra: Fluctuations, Academic Press, New York, (1956).

[39] H.A. Weidenmüller and G.E. Mitchell, Rev. of Mod. Phys. 81, 541 (2009).

[40] G.M. Mitchell, A. Richter and H.A. Weidenmüller, Rev. of Mod. Phys. 82, 2845 (2010).

[41] K. Riisager, Rev. of Mod. Phys. 66, 1105 (1994).

[42] P.G. Hansen, A.S. Jensen and B. Jonson, Ann. Rev. Nucl. Part. Sci. 45, 591 (1995).

[43] P.G. Thirolf and D. Habs, Prog. in Part. Nucl. Phys., 49, 325 (2002).

[44] C. Hugenschmidt, K. Schreckenbach, D. Habs and P.G. Thirolf, High-Intensity and High-Brilliance Sources of Moderated Positons Using a Brilliant $\gamma$ Beam arXiv-1103:0513v2[nucl-ex]2011, accepted by Appl. Phys. B. 
[45] D. Habs and U. Köster, Production of Medical Radioisotopes with High Specific Activity in Photonuclear Reactions with $\gamma$ Beams of High Intensity and Large Brilliance, arXiv-1008.5336v2[physics.med-ph]2010, accepted by Appl. Phys. B, DOI 10.1007/S00340-010-4278-1

[46] D. Habs et al., Medical Application Studies at ELI-NP, this SPIE conference (2011).

[47] T. Hayakawa et al., Nucl. Instr. Meth. A 261, 695 (2010).

[48] C.G. Schroer et al., Act. Phys. Pol. A 117, 357 (2010).

[49] D. Habs et al., Neutron Halo Isomers in Stable Nuclei and their Possible Application for the Production of Low Energy, Pulsed, Polarized Neutron Beams of High Intensity and High Brilliance, arXiv-1008.5324v1[nuclex]2010, accepted by Appl. Phys. B. 